\title{
Direct Calculation of the Turbulent Dissipation Efficiency in Anelastic Convection
}

\author{
Kaloyan Penev \\ 60 Garden St., M.S. 10, Cambridge, MA 02138 \\ Joseph Barranco \\ 1600 Holloway Avenue, San Francisco, CA 94132-4163 \\ Dimitar Sasselov \\ 60 Garden St., M.S. 16, Cambridge, MA 02138
}

Received — 


\begin{abstract}
The current understanding of the turbulent dissipation in stellar convective zones is based on the assumption that the turbulence follows Kolmogorov scaling. This assumption is valid for some cases in which the time frequency of the external shear is high (e.g. solar p-modes). However, for many cases of astrophysical interest (e.g. binary orbits, stellar pulsations etc.) the timescales of interest lie outside the regime of applicability of Kolmogorov scaling. We present direct calculations of the dissipation efficiency of the turbulent convective flow in this regime, using simulations of anelastic convection with external forcing. We show that the effects of the turbulent flow are well represented by an effective viscosity coefficient and we provide the values of the effective viscosity as a function of the perturbation frequency and compare our results to the perturbative method for finding the effective viscosity of Penev et al. (2008b) that can be applied to actual simulations of the surface convective zones of stars.
\end{abstract}

\title{
1. Introduction
}

For stars with surface convection, turbulent dissipation in the convective zone is believed to be the dominant mechanism responsible for the conversion of mechanical energy of tides, stellar oscillations and stellar pulsations to heat. Thus, this is the mechanism believed to determine the rates of tidal synchronization and circularization (Zahn 1966, 1989), the amplitudes of stellar $p$ modes (Goldreich \& Keeley 1977; Goldreich \& Kumar 1988; Goldreich et al. 1994), and the instability of stars to pulsations (Gonczi 1982).

The simplest approach to estimating the dissipation efficiency of the turbulent convection is to assume that the turbulence is homogeneous and isotropic and its interaction 
with external shear is only local. In that case, we can define an effective viscosity coefficient which will capture the effects due to the turbulent flow. The question then is how to find the value of this coefficient.

Currently two prescriptions, based on the assumption of Kolmogorov cascade, exist for estimating this coefficient as a function of the time period of the external shear $(T)$.

Zahn (1966, 1989) proposes that, the effective viscosity should scale linearly with the fraction of a turn eddies manage to complete in half a perturbation period:

$$
\nu=\nu_{\max } \min \left[\left(\frac{T}{2 \tau}\right), 1\right] .
$$

On the other hand, Goldreich \& Nicholson (1977) and Goldreich \& Keeley (1977), argue that eddies with turnover times bigger than $T / 2 \pi$ will not contribute to the dissipation. Then Kolmogorov scaling predicts:

$$
\nu=\nu_{\max } \min \left[\left(\frac{T}{2 \pi \tau}\right)^{2}, 1\right]
$$

Zahn's more efficient dissipation seems to be in better agreement with observations of tidal circularization times for binaries containing a giant star (Verbunt \& Phinney 1995), the location of the red edge of the Cepheid instability strip (Gonczi 1982), and even this more efficient prescription might be insufficient to explain the main sequence circularization of binary stars in clusters (Meibom \& Mathieu 2005).

However, Goldreich \& Keeley (1977), Goldreich \& Kumar (1988) and Goldreich et al. (1994), successfully used the less efficient dissipation to develop a theory for the damping of the solar $p$-modes. In this case the more effective dissipation would require dramatic changes in the excitation mechanism in order to explain the observed amplitudes.

Finally, Goodman \& Oh (1997) calculated a lowest order expansion of the effective viscosity, which when applied to Kolmogorov turbulence predicts a result closer to the less 
efficient Goldreich \& Nicholson viscosity. While this gives a firmer theoretical foundation for the quadratic prescription it does not help with the observational problem of insufficient dissipation in the case of tides and stellar pulsations.

A possible resolution of this problem is suggested by the fact that the successful applications of the two prescriptions correspond to very different perturbation periods. The Zahn (1966, 1989) scaling seems to work well for periods of order days, and the Goldreich and collaborators quadratic scaling seems to apply for periods of order minutes. This distinction is important because, in stars with surface convection, Kolmogorov scaling predicts that the eddies with turnover times of several minutes would have typical sizes that are very small compared to the local pressure scale height and any other external length scales. On the other hand turnover times of days correspond to eddies with typical sizes comparable or larger than the local pressure scale height. In this case Kolmogorov scaling is not expected to apply.

The flow seen in 2D and 3D simulations of stellar convection is very different from a Kolmogorov cascade (Sofia \& Chan 1984; Stein \& Nordlund 1989; Malagoli et al. 1990). There are two important distinctions. The first is that the velocity power spectrum is much flatter in the simulations than Kolmogorov, and so one expects to find a slower loss of dissipation efficiency with increased frequency of the external shear, assuming that the dissipation is dominated by the resonant eddies, as long as the external shear has a period that corresponds to eddy turnover times too long to fall in the inertial subrange of Kolmogorov turbulence. The second is that the flow is no longer isotropic and hence the effective viscosity should be a tensor, rather than a scalar.

As a first attempt to explore this possibility Penev et al. (2007, 2008b) adapted the Goodman \& Oh (1997) perturbative calculation to the Robinson et al. (2003) numerical model of stellar convection and found an asymmetric effective viscosity that scaled linearly 
with the period of the external shear. However, their perturbative calculation is applicable only as long as the forcing period $T$ is small compared to the turnover time of the largest eddies. In particular the perturbative treatment is not able to provide the maximum value the effective viscosity reaches and the frequency at which it reaches it, which is of great importance in calculating tidal interactions and dissipation of pulsations.

In this article we use the Penev et al. (2008a) spectral anelastic code to perform a direct calculation of the turbulent dissipation in a convective zone, by introducing external shear as an extra body force in the fluid equations. The goal is to investigate the applicability of effective viscosity as an approximation to the actual turbulent dissipation, and to derive directly an effective viscosity prescription and compare it against the Goodman \& Oh (1997) formalism.

\section{Simulations}

\subsection{Steady State Convection}

The details of the numerical simulation and the equations evolved are presented in Penev et al. (2008a). We are simulating a rectangular box with impenetrable, constant temperature top and bottom boundaries (the $\hat{z}$ velocity vanishes and the temperature is held at some constant value at the top and bottom walls of the box) using the anelastic approximation.

The background state, and the parameters with which all the runs presented in this paper were computed, are the same as the parameters used for all convectively unstable tests of section 4.1 of Penev et al. (2008a). For convenience we remind them here: 


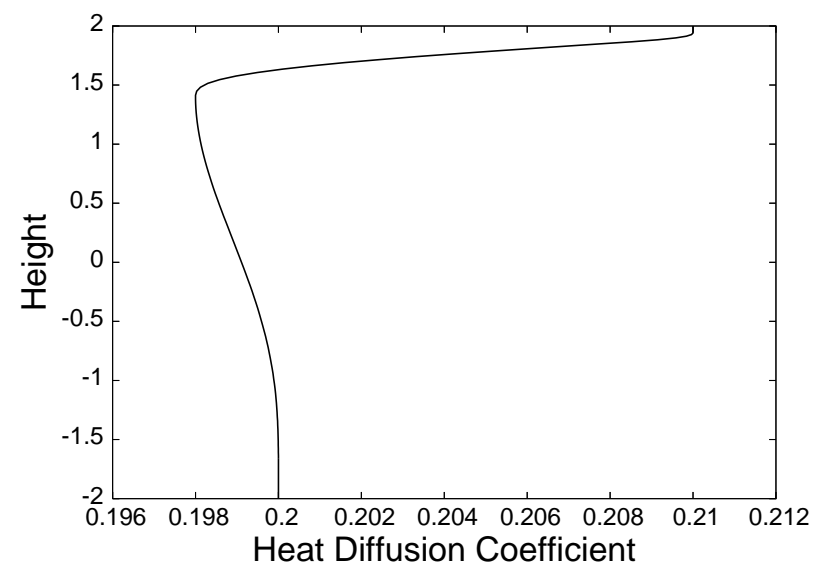

Fig. 1.- The height dependent heat diffusion coefficient

$$
\begin{array}{cl}
L_{x}=L_{y}=L_{z}=4 & \text { physical dimensions of the convective box. } \\
N_{x}=N_{y}=N_{z}=128 & \text { resolution in each direction } \\
p_{\text {top }}=1.0 \times 10^{5} & \text { background pressure at the top of the box } \\
g=2.74 & \text { gravitational acceleration, in }-\hat{z} \text { direction } \\
C_{p}=0.21 & \text { specific heat at constant pressure of the fluid } \\
R=8.317 \times 10^{-2} & \text { ideal gas constant of the fluid } \\
T_{\text {low }}=10.0 & \text { temperature at the top boundary of the box } \\
T_{\text {high }}=62.37 & \text { temperature at the bottom boundary of the box }
\end{array}
$$

The vertical profile of the heat diffusion coefficient we imposed is presented in figure 1 .

We initialize the box with random entropy fluctuations and let it evolve with time until a steady state is reached. The criteria we used for concluding a steady state has been reached were, that the kinetic and thermal energies should stop drifting systematically, and only exhibit oscillations at the approximate convective turnover time (see fig. 2), and that the spatial spectra of the velocity and potential temperature remain constant to within a few percent. The steady state Fourier spatial and time spectra are presented in fig. 3. 

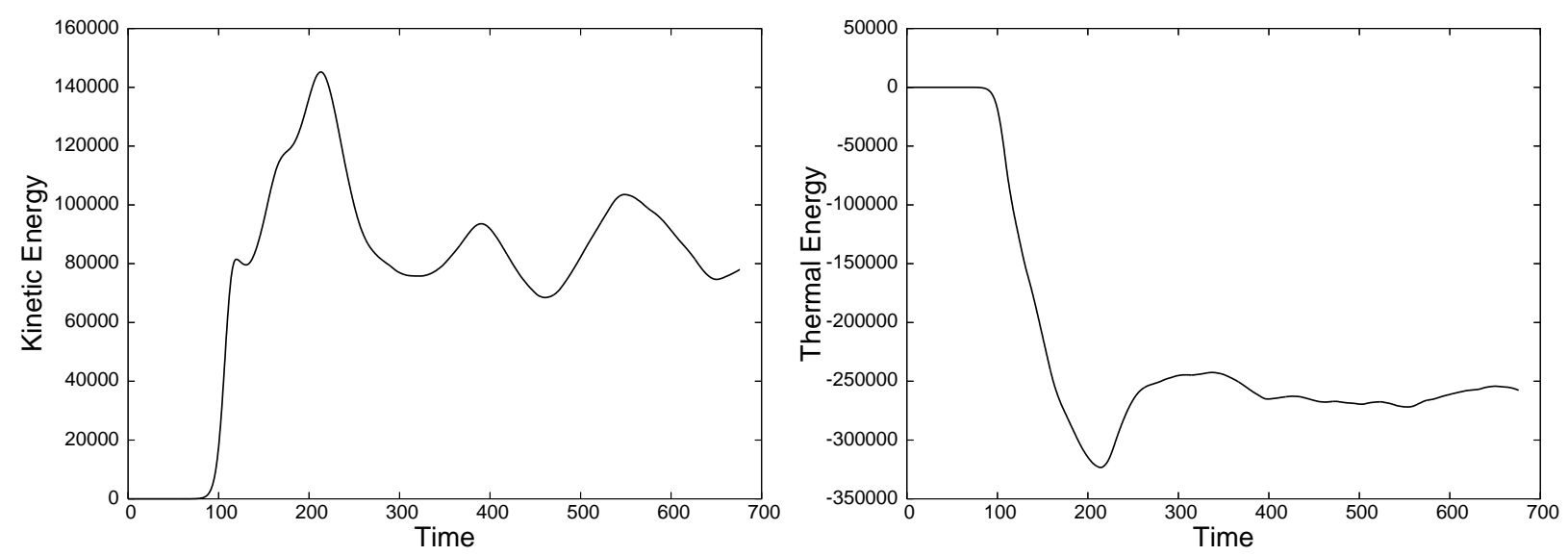

Fig. 2.- Kinetic (left) and thermal (right) energy content of the convective box used as one of the criteria for having reached a steady state. We decided steady state was reached for times greater than 400 .

\subsection{External Forcing}

After steady state has been reached we introduce external forcing $(\mathbf{f})$ in the form of a position and time dependent gravitational acceleration in addition to the already present vertical gravity. So, in short, the anelastic momentum equation that we evolve is:

$$
\frac{\partial \mathbf{v}}{\partial t}=\mathbf{v} \times \omega-\nabla \widetilde{h}+\frac{\tilde{\theta}}{\bar{\theta}} g \hat{\mathbf{z}}+\mathbf{f},
$$

where quantities with tilde represent anelastic perturbations to the background variable (denoted by an over bar), $\mathbf{v}$ is the velocity vector, $\widetilde{h} \equiv \widetilde{p} / \bar{\rho}+\mathbf{v}^{2} / 2$ is the enthalpy, $\widetilde{\theta}$ is the perturbation to the background potential temperature, $\bar{\theta}=\bar{T}\left(p_{0} / \bar{p}\right)^{R / C_{p}}$, and $\mathbf{f}$ is the external forcing. We have investigated the effects of two forms of forcing:

1. $Z$ (height) dependent horizontal forcing with a Gaussian profile:

$$
\mathbf{f}(z, t)=f_{0} e^{-2 z^{2}} \cos \left(\frac{2 \pi t}{T}\right) \hat{\mathbf{x}} .
$$





Fig. 3.- The (x: top left, y: top right, z: bottom left, time: bottom right) spectra of the 3 velocity components and the potential temperature. The thick line in the spatial spectra plots corresponds to Kolmogorov scaling $\left(E_{k} \propto k^{-5 / 3}\right)$. The thick line in the time spectra plot corresponds to the scaling Penev et al. (2008a) found for the effective viscosity. 
2. $Y$ dependent forcing in the $\hat{x}$ direction, again with a Gaussian profile:

$$
\mathbf{f}(y)=f_{0} e^{-2 y^{2}} \cos \left(\frac{2 \pi t}{T}\right) \hat{\mathbf{x}}
$$

Clearly the amplitude of the forced velocity will be approximately $f_{0} T / 2 \pi$, so in order to investigate the period dependence of the dissipation, we simulated a number of flows with different periods and the same $f_{0} T$. That way, the shear created by the external forcing was the same for all the flows in the set. We computed two such sets for each forcing case: one with $f_{0} T=1$ and another with $f_{0} T=0.15$. In addition we performed another set of simulations with fixed period and varying values of $f_{0} T$ in order to investigate the effects of the forcing amplitude on the dissipation efficiency. Appendix ?? summarizes the runs and the respective number of time steps simulated for each case.

Notice that the weak forcing cases require a lot more time steps in order to average out the turbulent noise and allow us to detect the systematic energy dissipation. Similarly, the $y$ dependent forcing requires longer runs as well, because in this case, for the same forcing there is less dissipation due to the anisotropic effective viscosity.

For the strong forcing case, we expect the maximal central velocities to reach $\max v_{x}=1 / 2 \pi \approx 0.16$, and for the weak forcing $\max v_{x}=0.15 / 2 \pi \approx 0.024$. For comparison, in our convective box the speed of sound varies between 1.2 at the top of the box and 2.9 at the bottom and the typical r.m.s. velocities are between 0.02 and 0.04 (see figure 4), except very near the top and bottom of the box where the collision of the vertical flow with the impenetrable boundaries results in higher horizontal velocities.

In the case of tides in binary stellar systems, the velocities excited by the external forcing are small compared to the typical convective velocities, however, performing numerical simulations with forcing small enough to ensure that this holds will be prohibitive in terms of computational time, because it will require simulations for excessive number 

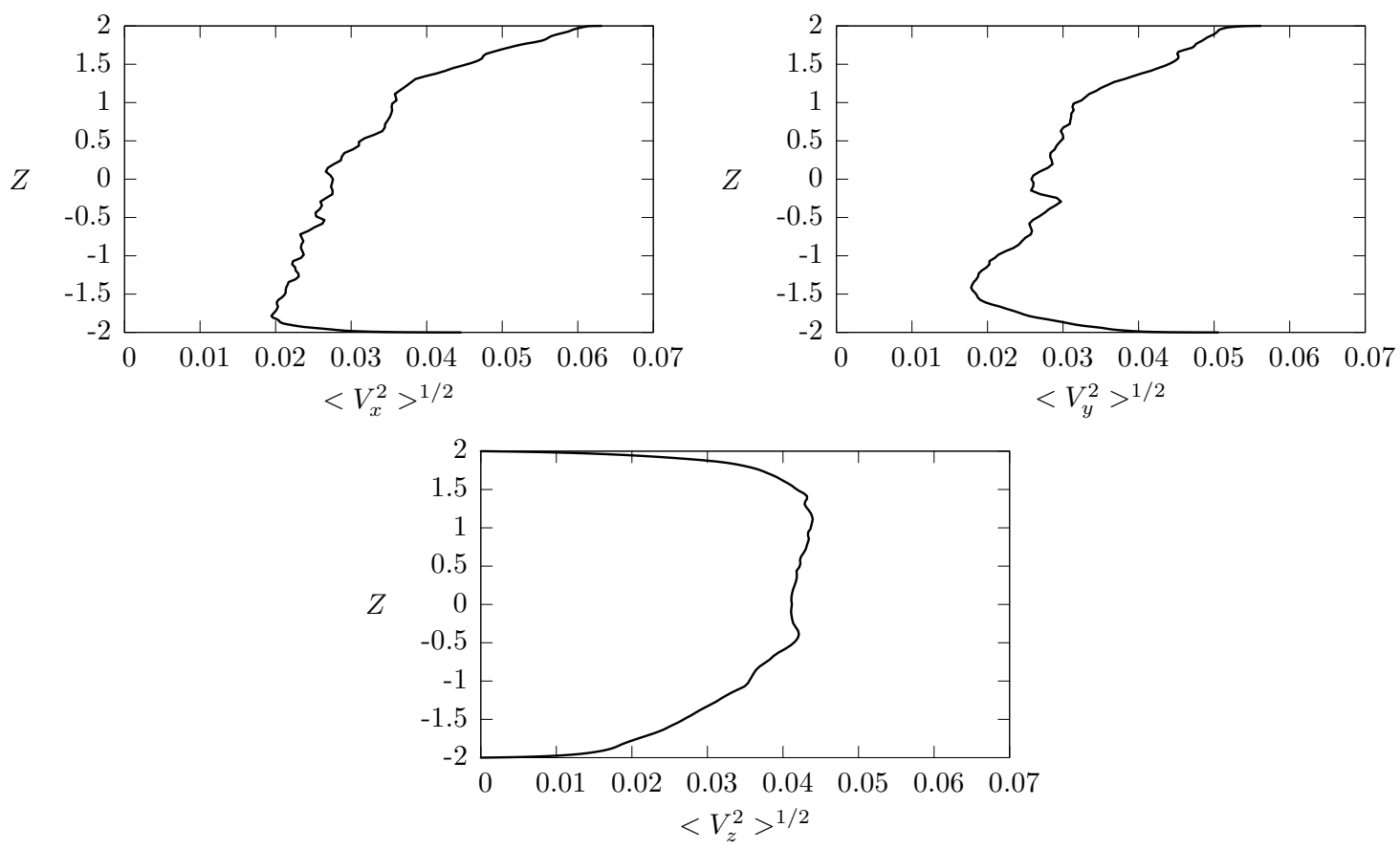

Fig. 4. - The typical steady state r.m.s. velocities ( $v_{x}$ top left, $v_{y}$ top right, $v_{z}$ bottom) in the absence of forcing.

of time steps to make sure the dissipation is noticeable among the fluctuations due to the turbulence (see section 3.2).

\section{Results}

\subsection{Anisotropic Viscosity}

The viscosity in this work, as in Penev et al. (2008a) is described by a rank-4 tensor $\left(K_{i j m n}\right)$, which gives the relation between the strain rate $e_{i j} \equiv 1 / 2\left(\partial_{i} v_{j}+\partial_{j} v_{i}\right)$ and the viscous stress $\sigma_{i j}$ :

$$
\sigma_{i j}=K_{i j m n} e_{m n}
$$


where summation over repeated indices is assumed.

This tensor must obey a set of symmetries in order to avoid infinite torques. In addition we expect it to be symmetric with respect of rotations and reflections around the $\hat{z}$ axis. With all these symmetries $K_{i j m n}$ can be shown to have only six independent components. Of those, the particular forms of forcing described in section 2.2 probe only two families:

$$
\left.\begin{array}{r}
K_{1221}=K_{2121}=K_{2112}=K_{1212} \\
K_{3131}=K_{3113}=K_{1331}=K_{2323} \\
K_{3232}=K_{3223}=K_{2332}
\end{array}\right\}=K_{1313},
$$

which will be referred to as $K_{1212}$ and $K_{1313}$ from now on.

\subsection{Direct Viscosity Calculation}

To simplify the discussion we define upfront the following quantities for the $z$ dependent forcing:

$$
\begin{aligned}
C_{x z}(z) & =\sum_{i, j, w} v_{x}\left(x_{i}, y_{j}, z, t_{w}\right) \cos \left(\frac{2 \pi t_{w}}{T}\right), \\
\text { and } S_{x z}(z) & =\sum_{i, j, w} v_{x}\left(x_{i}, y_{j}, z, t_{w}\right) \sin \left(\frac{2 \pi t_{w}}{T}\right),
\end{aligned}
$$

and for the $y$ dependent forcing:

$$
\begin{aligned}
C_{x y}(y, z) & =\sum_{i, w} v_{x}\left(x_{i}, y, z, t_{w}\right) \cos \left(\frac{2 \pi t_{w}}{T}\right), \\
\text { and } \quad S_{x y}(y, z) & =\sum_{i, w} v_{x}\left(x_{i}, y, z, t_{w}\right) \sin \left(\frac{2 \pi t_{w}}{T}\right)
\end{aligned}
$$

where $x_{i}$ and $y_{j}$ are the locations of the $x$ and $y$ collocation grid points, and $t_{w}$ are the times at which we have sampled the velocity field. We evaluate the sum over an integer number of forcing periods $T$. 


\subsubsection{Depth Dependence of the Effective Viscosity}

We use pre-determined functions of depth, up to a normalization constant, for the effective viscosity coefficients that we need, namely:

$$
\begin{aligned}
K_{1313} & =K_{1313}^{0}\left\langle v_{z}^{2}\right\rangle^{1 / 2} H_{p} \\
\text { and } K_{1212} & =K_{1212}^{0}\left\langle\frac{v_{x}^{2}+v_{y}^{2}}{2}\right\rangle^{1 / 2} H_{p} .
\end{aligned}
$$

This is the same scaling that we used in presenting the perturbative result ?, with the only difference that only the velocity in the direction of the external shear is used as the velocity scale. In equation 13 we average together both horizontal components of the velocity, because on average there should be no physical difference between the two. In practice, the different velocity scaling makes little difference, since as we can see from fig. 4, away from the boundaries all components of the velocity behave alike, except for the fact that $v_{z}$ tends to be larger. So, using the full r.m.s. velocity instead of only one component, just leads to smaller values of the normalization constants $K_{1313}^{0}$ and $K_{1212}^{0}$.

\subsubsection{Fitting the Spatial Dependence of the Dissipation}

We would like to verify the applicability of the effective viscosity framework to the problem of turbulent dissipation, by showing that substituting the turbulent flow with a simple viscosity is able to capture not only the total amount of energy dissipated, but also the momentum transport, or in other words the spatial dependence of this dissipation.

For the $z$ dependent $x$ forcing, we would like to show that the work per unit mass done by the forcing on the flow at each depth:

$$
W_{x z}^{\text {turb }}(z) \equiv f_{0} e^{-2 z^{2}} C_{x z}(z)
$$

matches the energy that would be transported and dissipated out of that depth by an 
assumed effective viscosity:

$$
\begin{aligned}
W_{x z}^{v i s c}(z) \equiv & \frac{1}{2} K_{1313}(z)\left[C_{x z}(z) C_{x z}^{\prime \prime}(z)+S_{x z}(z) S_{x z}^{\prime \prime}(z)\right]+ \\
& +\frac{1}{2}\left(K_{1313}^{\prime}(z)+\frac{d \ln \bar{\rho}}{d z} K_{1313}(z)\right)\left[C_{x z}(z) C_{x z}^{\prime}(z)+S_{x z}(z) S_{x z}^{\prime}(z)\right],
\end{aligned}
$$

where primes denote derivatives with respect to $z$ and $\bar{\rho}$ is the background density profile.

For the $y$ dependent $x$ forcing, we would like to show that the work per unit mass done by the forcing on the flow at each $y$ plane:

$$
W_{x y}^{t u r b}(y) \equiv \frac{f_{0}}{N} e^{-2 y^{2}} \int_{z_{\min }}^{z_{\max }} \bar{\rho}(z) C_{x y}(y, z) d z
$$

matches the energy that would be transported and dissipated out of that plane by an assumed effective viscosity:

$$
W_{x y}^{v i s c}(y) \equiv \frac{1}{2 N} \int_{z_{\min }}^{z_{\max }} \bar{\rho}(z) K_{1212}(z)\left[C_{x y}(y, z) C_{x y}^{\prime \prime}(y, z)+S_{x y}(y, z) S_{x y}^{\prime \prime}(y, z)\right] d z
$$

where now primes denote derivatives with respect to $y, z_{\min }$ and $z_{\max }$ are the minimal and maximal depth respectively that we want to include in the fit and $N \equiv \int_{z_{\min }}^{z_{\max }} \bar{\rho}(z) d z$. The reason we do not want to include the entire simulated domain is that near the boundaries the flow is strongly affected by the impenetrable top and bottom walls and is thus non-physical.

We find the values of $K_{1313}^{0}$ and $K_{1212}^{0}$ by least squares fitting of $W_{x z}^{\text {visc }}$ to $W_{x z}^{\text {turb }}$ in the range $z_{\min }<z<z_{\max }$ and $W_{x y}^{v i s c}$ to $W_{x y}^{\text {turb }}$ in the range $-L_{y} / 2<y<L_{y} / 2$ respectively. Clearly, the presence of the turbulence will cause random fluctuations in the velocity profile which should average out if we combine a large enough number of time steps in evaluating the quantities $C_{x z}, C_{x y}, S_{x z}$ and $S_{x y}$. These fluctuations are highly amplified when we estimate second derivatives of those quantities, so $W_{x z}^{v i s c}$ and $W_{x y}^{v i s c}$ suffer much more than $W_{x z}^{t u r b}$ and $W_{x y}^{t u r b}$. Some representative fitted curves for each series of runs are shown in figures 5, 6] and 7. 

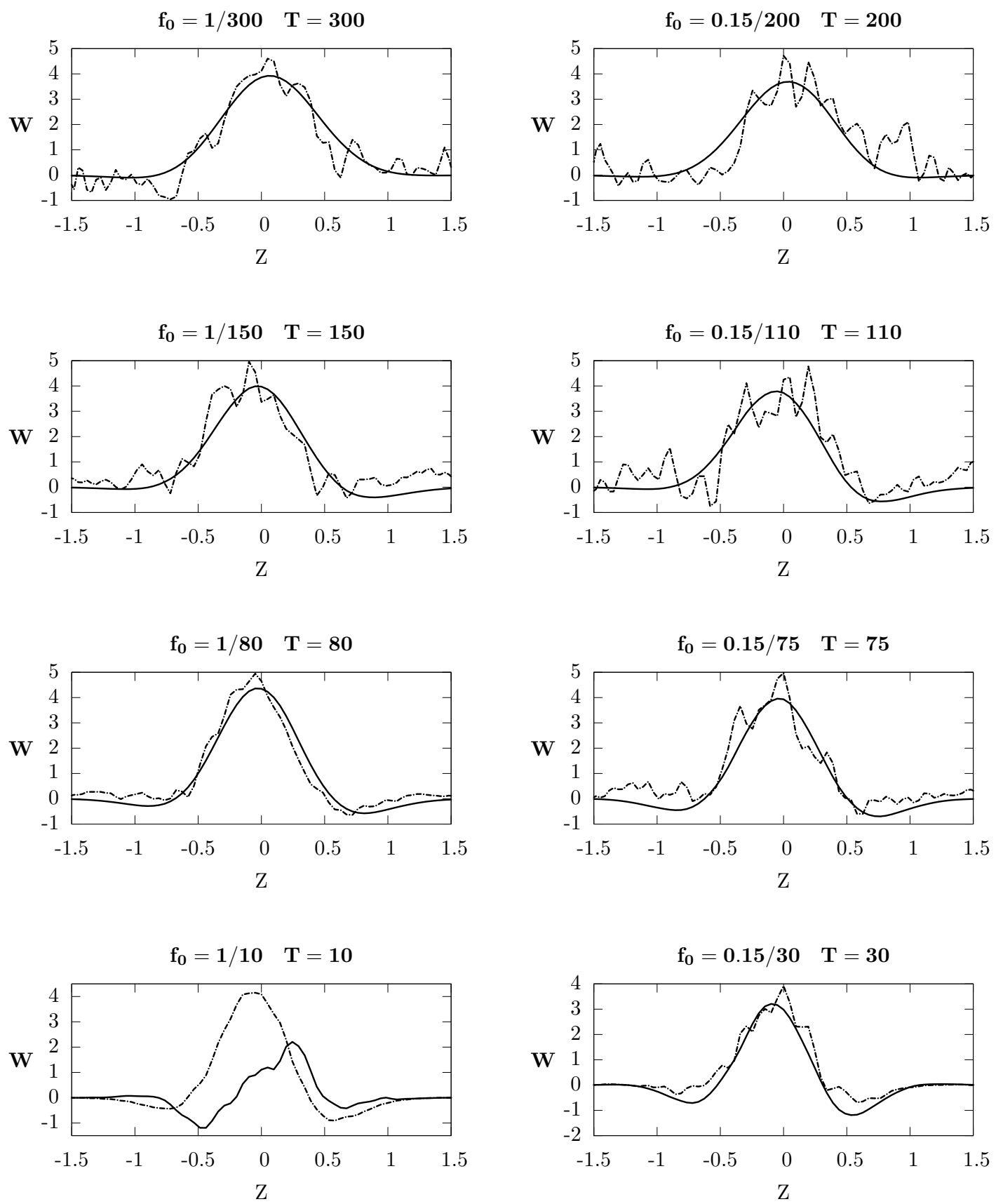

Fig. 5.- Typical least squares fits between $W_{x z}^{\text {turb }}$ (solid curves) and $W_{x z}^{v i s c}$ (dashed curves) for the two sets of simulations with $z$ dependent $x$ forcing with fixed amplitude and variable period. Left: strong forcing, right: weak forcing. In each plot $W_{x z}^{\text {turb }}$ and $W_{x z}^{v i s c}$ have been scaled in order to make their maximum values be of order few. 

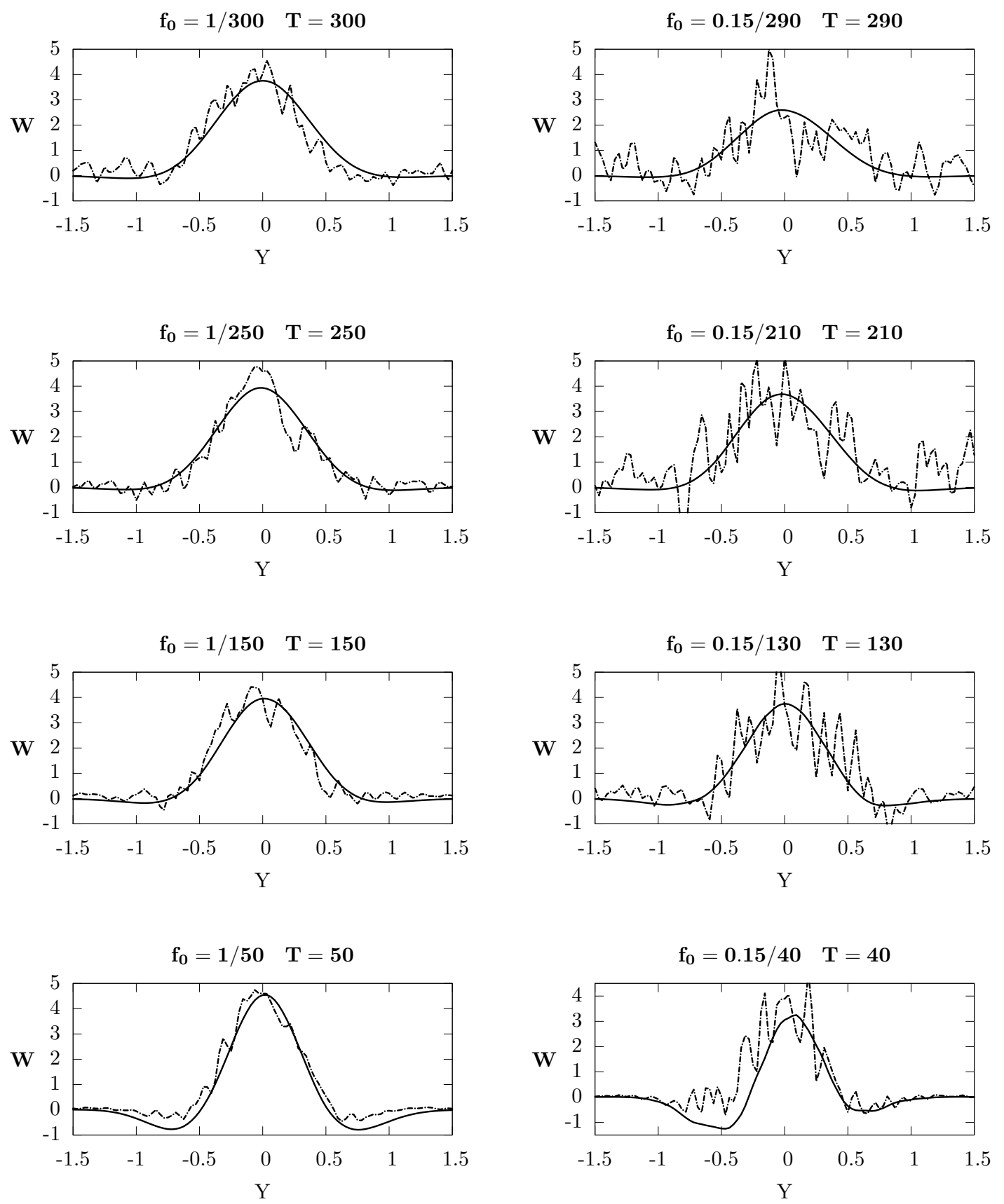

Fig. 6. - Typical least squares fits between $W_{x y}^{\text {turb }}$ (solid curves) and $W_{x y}^{v i s c}$ (dashed curves) for the two sets of simulations with $y$ dependent $x$ forcing with fixed amplitude and variable period. Left: strong forcing, right: weak forcing. In each plot $W_{x y}^{t u r b}$ and $W_{x y}^{v i s c}$ have been scaled in order to make their maximum values be of order few. 

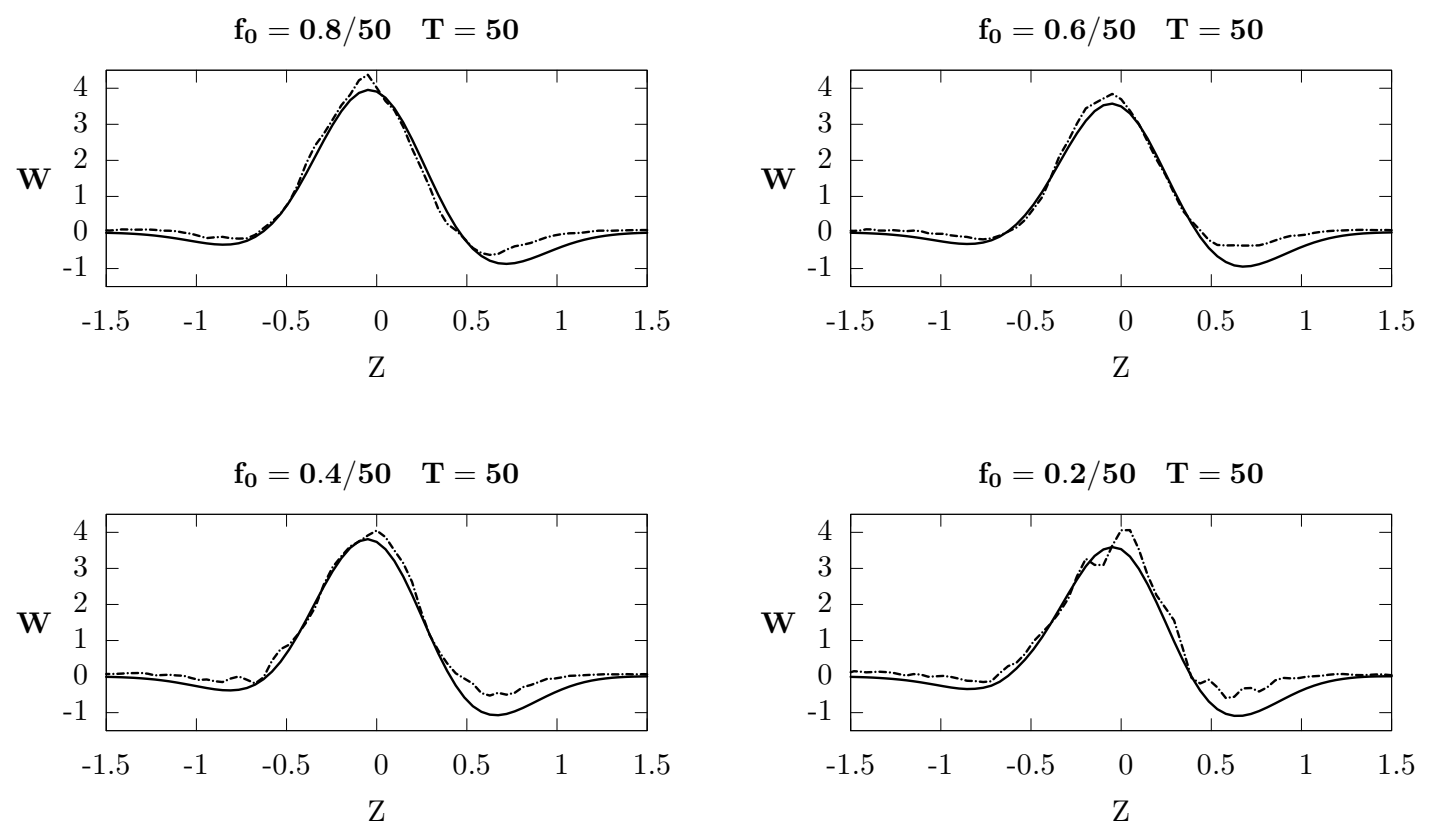

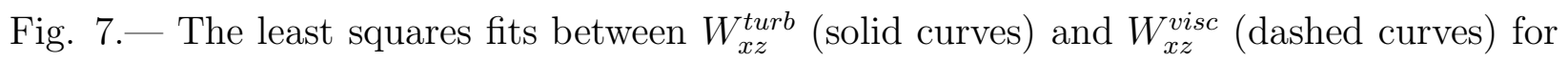
the set of simulations with $z$ dependent $x$ forcing with fixed period and variable amplitude. In each plot $W_{x z}^{t u r b}$ and $W_{x z}^{v i s c}$ have been scaled in order to make their maximum values be of order few. 
We see that, except for one case, the two curves match closely, which shows that the effective viscosity assumption captures the effects of turbulent dissipation to a good degree. The $T=10$ curves for the strong $z$ dependent $x$ forcing case do not match well at all. The bad fit is due to the fact that if there is any dissipation present it is undetectable from within the turbulent noise.

The reason for the strongly suppressed dissipation is that for such short periods the corresponding eddy sizes are too small to be reliably simulated at our current resolution. This fact points to the Goldreich et. al. picture, that the dissipation is dominated by eddies with periods close to close that of the external forcing $(T)$, as opposed to the Zahn picture in which the largest eddies are always the most important, since in the latter case we would expect the linear scaling to continue to arbitrarily short periods.

\subsubsection{Matching Deposited to Dissipated Power}

An alternative way to get a value for the scaling constants $K_{1313}^{0}$ and $K_{1212}^{0}$ is to equate the overall power deposited into the box by the external forcing:

$$
\begin{aligned}
& \dot{\varepsilon}_{x z}^{e x t}=\int_{-z_{\min }}^{z_{\max }} \bar{\rho}(z) W_{x z}^{t u r b}(z) d z \quad, \text { for } \quad K_{1313}^{0} \\
& \dot{\varepsilon}_{x y}^{e x t}=N \int_{-L_{y} / 2}^{L_{y} / 2} W_{x y}^{t u r b}(y) d y \quad, \text { for } \quad K_{1212}^{0} \text {, }
\end{aligned}
$$

to the value that the effective viscosity dissipates out of the box:

$$
\begin{array}{ccc}
\dot{\varepsilon}_{x z}^{v i s c}=\int_{-z_{\min }}^{z_{\max }} \bar{\rho}(z) \widetilde{W}_{x z}^{v i s c}(z) d z & \text { for } & K_{1313}^{0}, \\
\dot{\varepsilon}_{x y}^{v i s c}=\int_{-L_{y} / 2}^{L_{y} / 2} \widetilde{W}_{x y}^{v i s c}(y) d y & \text { for } & K_{1212}^{0}
\end{array}
$$


Where we have defined:

$$
\begin{aligned}
& \widetilde{W}_{x z}^{v i s c}(z) \equiv K_{1313}(z)\left[\left(\frac{d C_{x z}}{d z}\right)^{2}+\left(\frac{d S_{x z}}{d z}\right)^{2}+\right] \\
& \widetilde{W}_{x y}^{v i s c}(y) \equiv \int_{z_{\min }}^{z_{\max }} \bar{\rho}(z) K_{1212}(z)\left[\left(\frac{\partial C_{x y}}{\partial y}\right)^{2}+\left(\frac{\partial S_{x y}}{\partial y}\right)^{2}\right] d z .
\end{aligned}
$$

Note that in this case we do not expect to match the spatial dependence, only the overall rate. The reason for this, is that viscous forces redistribute the energy in the box as well as dissipate it. So we cannot use these quantities to examine the applicability of the effective viscosity assumption.

Because evaluating $\widetilde{W}_{x z}^{v i s c}(z)$ and $\widetilde{W}_{x y}^{v i s c}(y)$ requires only first derivatives of the sine and cosine velocity components, they suffer significantly less from the turbulent noise than $W_{x z}^{v i s c}(z)$ and $W_{x y}^{v i s c}(y)$ from section 3.2.2. On the other hand, some energy transfer inevitably occurs near the top and bottom boundaries, which is excluded from evaluating the $z$ integrals. For the case of $z$ dependent forcing this is a small amount, since the forcing in that part of the box is very small by design. However, for the $y$ dependent forcing significant energy transfer does occur in those regions, which could bias the estimated viscosities toward lower values (see the last paragraph of section [3.3).

Plots of $\widetilde{W}_{x z}^{v i s c}(z)$ and $\widetilde{W_{x y}^{v i s c}}(y)$ for the same cases as in figures 5, 6] and 7 are shown in figures 8, 9] and 10 respectively, where we have taken $z_{\max }=-z_{\min }=1.5$ for the depth dependent forcing and $z_{\max }=-z_{\min }=1.8$ for the $y$ dependent forcing.

\subsection{Comparison Between the Perturbative and Direct Calculation}

We compare the Penev et al. (2008a) perturbative estimate of the $K_{1313}$ and $K_{1212}$ viscosity coefficients to the directly calculated effective viscosity, obtained by the procedures of section 3.2 , in figures 11 and 12 . 



Fig. 8. - The energy dissipation rate due to the effective viscosity at each depth for the two sets of simulations with $z$ dependent $x$ forcing with fixed amplitude and variable period. Left: strong forcing, right: weak forcing. The plots are for the same simulations as the plots in figure 5. The same scaling has been applied to each plot as in figure 5 

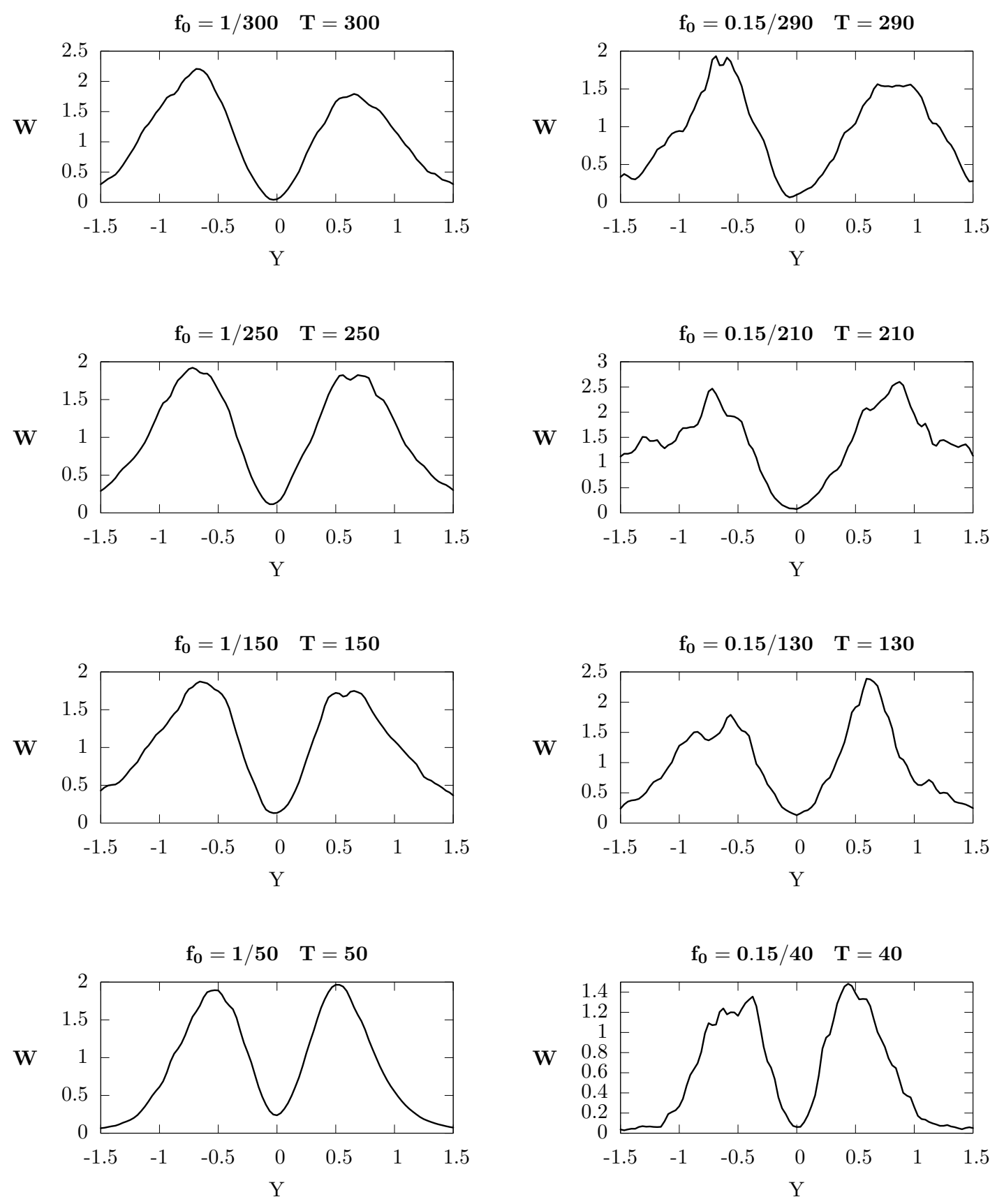

Fig. 9.- The energy dissipation rate due to the effective viscosity at each y plane for the two sets of simulations with $y$ dependent $x$ forcing with fixed amplitude and variable period. Left: strong forcing, right: weak forcing. The plots are for the same simulations as the plots in figure 6. The same scaling has been applied to each plot as in figure 6 

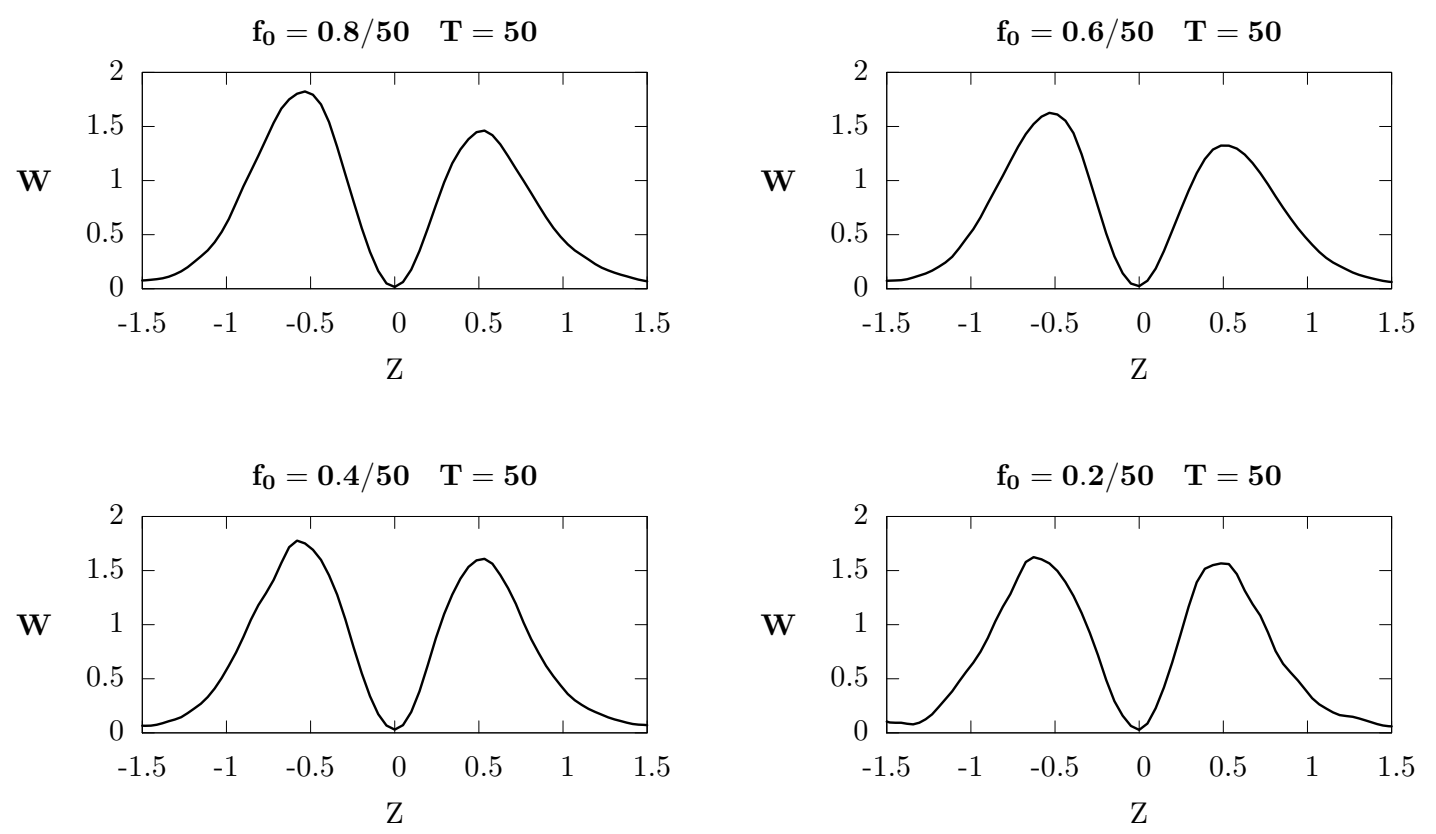

Fig. 10.- The energy dissipation rate due to the effective viscosity at each depth for the set of simulations with $z$ dependent $x$ forcing with fixed period and variable amplitude. The plots are for the same simulations as the plots in figure 7 The same scaling has been applied to each plot as in figure 7 

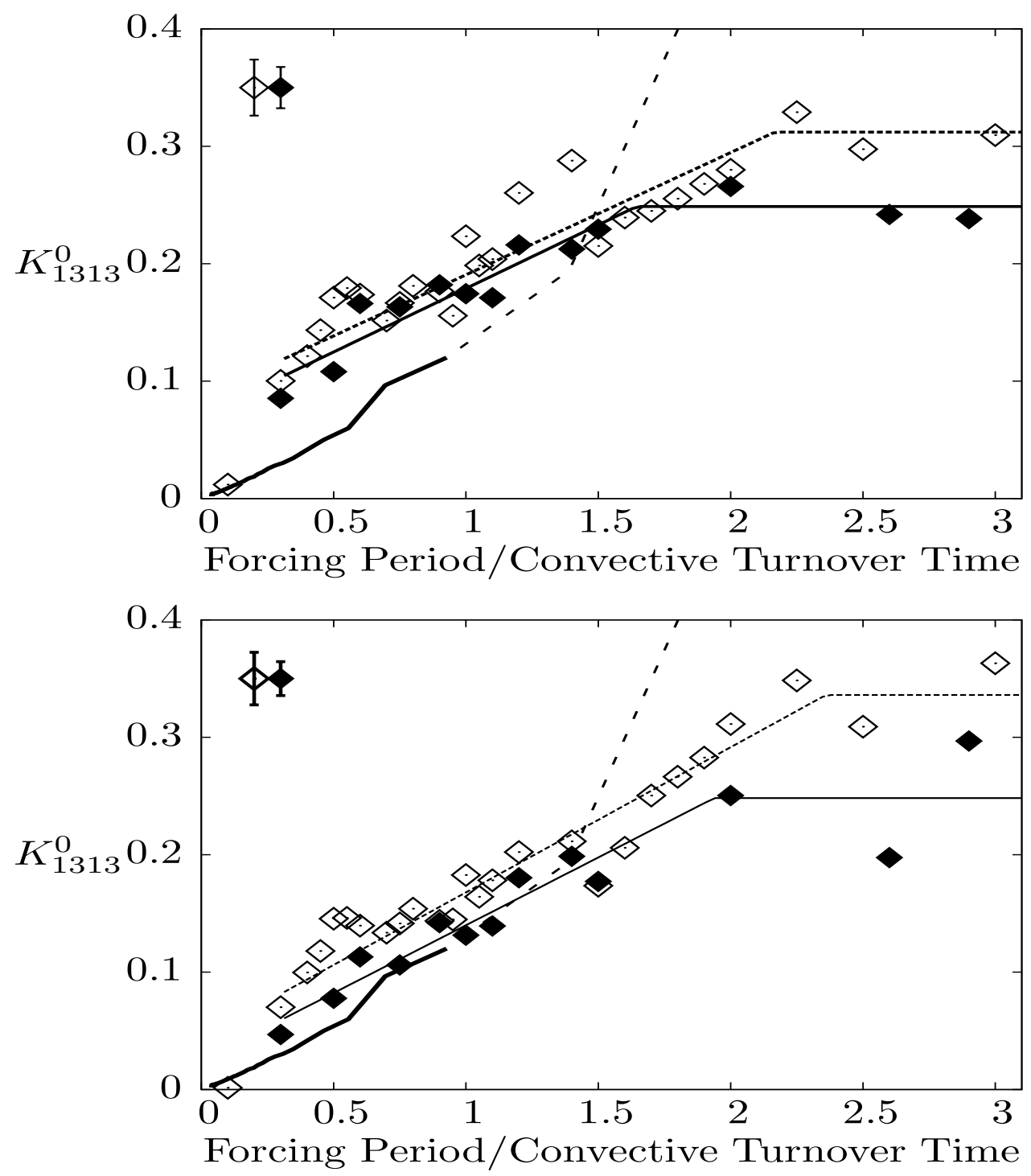

Fig. 11. - Comparison between the after-the-fact perturbatively estimated $K_{1313}^{0}$ (curve) and the viscosity obtained from the direct simulations by least squares fit of $W_{x z}^{v i s c}$ (equation 15) to $W_{x z}^{\text {turb }}$ (equation 14) - top ; and from setting $\int W_{x z}^{\text {turb }}(z)=\int \widetilde{W}_{x z}^{\text {visc }}(z)$ - bottom. The strong forcing points are plotted with empty symbols, and the weak forcing with filled symbols. The horizontal axis is the perturbation period $(T)$ in units of the convective turnover time in the box. Also shown are least squares fits to the strong forcing points (dashed line) and the weak forcing points (solid line), by a linear function with saturation. The error bars in the top left corner of the plots correspond to the standard deviation of $K_{1313}^{0}$ (assumed the same for all points) obtained from the differences with the fitted curves. 

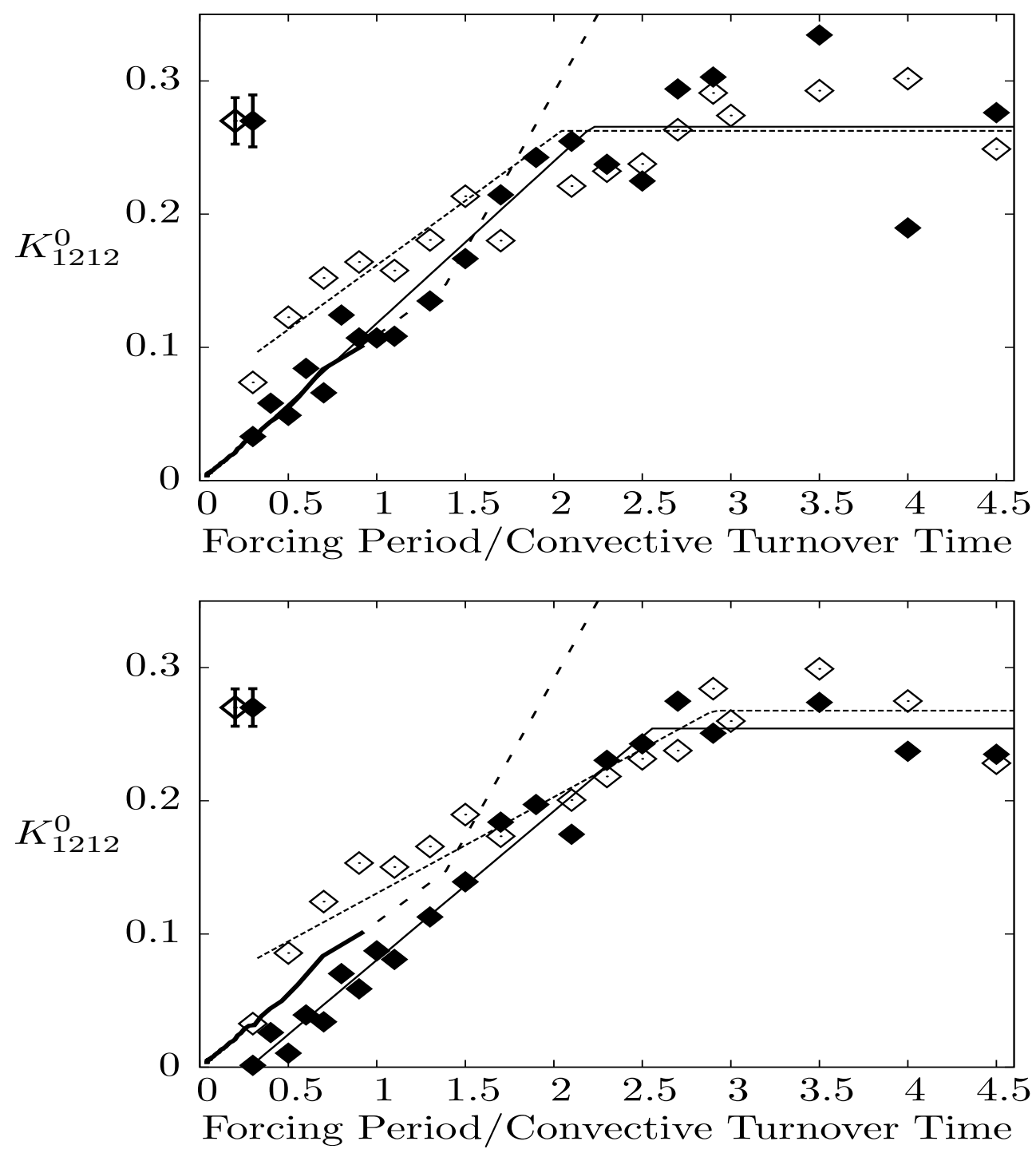

Fig. 12.- Comparison between the after-the-fact perturbatively estimated $K_{1212}^{0}$ (curve) and the viscosity obtained from the direct simulations by least squares fit of $W_{x y}^{\text {visc }}$ (equation 17) to $W_{x y}^{\text {turb }}$ (equation [16) - top ; and from setting $\int W_{x y}^{\text {turb }}(y)=\int \widetilde{W}_{x y}^{\text {visc }}(y)$ - bottom. The strong forcing points are plotted with empty symbols, and the weak forcing with filled symbols. The horizontal axis is the perturbation period $(T)$ in units of the convective turnover time in the box. Also shown are least squares fits to the strong forcing points (dashed line) and the weak forcing points (solid line) by a linear function with saturation. The error bars in the top left corner of the plots correspond to the standard deviation of $K_{1212}^{0}$ (assumed the same for all points) obtained from the differences with the fitted curves. 
The curves in those figures correspond to the perturbative expansion, and the points correspond to the direct calculation. Since the perturbative calculation assumes that the forcing period is small compared to the convective turnover time, we have used a solid line only for $T<\tau_{c}$. For longer periods (dotted line) this method is certainly not applicable.

We see that the effective viscosity predicted with all three methods scales linearly with the forcing period for most of the range covered by our simulations. Further, the direct calculations show that the effective viscosity saturates for long periods, after which it remains roughly constant. One physically expects to see this saturation, because for forcing periods much larger than any convective timescale there is no reason why the dissipation efficiency of the convective zone should depend on the period. The exact saturation period varies between the different forcing cases and even viscosity estimation methods, but it is seen to be somewhere in the range $1.5 \tau_{c}<T_{\text {sat }}<3 \tau_{c}$.

In order to obtain a functional dependence for the effective viscosity on period we perform a least square fit to the direct calculation points, where we fit a linear function that saturates at some period. The resulting fits are shown in figures 11 and 12 as solid lines for the weak forcing cases and dashed lines for the strong forcing cases. The parameters of the fitted lines are given in tables 1 and 2. This also allows us to get an estimate of the error bars associated with the points. Those are shown in the upper left corners of the plots in figures 11 and 12 .

Since for very short periods the perturbative calculation should be valid, and the turbulence should be well approximated by a Kolmogorov cascade, we expect that the scaling of the effective viscosity with period should be quadratic for those short periods. The proportionality constant for this steeper scaling should determine the zero crossings of the best fit linear approximations to the effective viscosity. From tables 1 and 2 we see that these are mostly positive, except for $K_{1212}^{0}$ in the weak forcing case, where for the fitting 
Table 1: The linear regression parameters corresponding to the solid and dashed lines in fig. 11 .

\begin{tabular}{lcc|cc} 
& \multicolumn{2}{c|}{ Depth Fit } & \multicolumn{2}{c}{ Dissipation Match } \\
& strong forcing & weak forcing & strong forcing & weak forcing \\
\hline slope & $0.10 \pm 0.012$ & $0.11 \pm 0.015$ & $0.12 \pm 0.009$ & $0.11 \pm 0.02$ \\
zero crossing & $0.086 \pm 0.013$ & $0.071 \pm 0.014$ & $0.044 \pm 0.011$ & $0.02 \pm 0.02$ \\
saturation period & $2.2 \pm 0.2$ & $1.64 \pm 0.15$ & $2.4 \pm 0.2$ & $2.0 \pm 0.2$
\end{tabular}

Table 2: The linear regression parameters corresponding to the solid and dashed lines in fig. 12 ,

\begin{tabular}{lcc|cc} 
& \multicolumn{2}{c|}{ Depth Fit } & \multicolumn{2}{c}{ Dissipation Match } \\
& strong forcing & weak forcing & strong forcing & weak forcing \\
\hline slope & $0.097 \pm 0.023$ & $0.12 \pm 0.02$ & $0.073 \pm 0.023$ & $0.11 \pm 0.01$ \\
zero crossing & $0.065 \pm 0.023$ & $-0.003 \pm 0.023$ & $0.058 \pm 0.014$ & $-0.03 \pm 0.008$ \\
saturation period & $2.0 \pm 0.3$ & $2.2 \pm 0.2$ & $2.9 \pm 0.2$ & $2.6 \pm 0.1$
\end{tabular}

method the zero crossing is basically indistinguishable from zero, and in the dissipation matching method it is slightly negative.

We believe that this negative zero crossing is caused by the fact that the forcing in this case is not small near the boundaries, and as a result significant energy is deposited there by the external forcing, especially since in those regions the horizontal velocity is very large, due to the collision of the mainly vertical flow in the bulk of the box with the impenetrable boundaries. This large horizontal flow is non physical and as such we do not include the regions near the boundaries in our calculations. Ignoring this external energy source is not important for the fitting of the spatial dependence of the deposited energy since it will be ignored from both $W_{x y}^{\text {turb }}$ and $W_{x y}^{\text {visc }}$. However, if some amount of this energy makes it to 
the region of the box used to calculate $\widetilde{W}_{x y}^{v i s c}$ before the turbulent cascade has dissipated it, it will act to artificially increase $\widetilde{W}_{x y}^{v i s c}$, and consequently decrease the effective viscosity we calculate.

\subsubsection{Amplitude Dependence}

The three dependences in each of the plots of figures 11 and 12 correspond to three different forcing strengths: strong forcing with $v_{\text {forc }} / v_{\text {conv }} \approx 2.7$, weak forcing with $v_{\text {forc }} / v_{\text {conv }} \approx 0.4$ and the pertubative calculation with $v_{\text {forc }} / v_{\text {conv }} \ll 1$, where $v_{\text {forc }}$ is the peak velocity due to the external forcing and $v_{c o n v}$ is the root mean square velocity for the central plane of the box in the absence of forcing.

The systematic difference between the three viscosities suggests a possibly important amplitude dependence of the dissipation efficiency. In order to get some idea for the importance of the magnitude of the external shear in determining the values of $K_{1313}^{0}$ we performed four additional simulations with $z$ dependent external forcing with period $T=\tau_{c} / 2$ with strengths intermediate between the strong and weak forcing cases considered above (see table ?? for the details of each run and figures 7 and 10 for the energy rate curves). Using the same two methods discussed above, we estimate the effective viscosity for those cases, which we plot in figure 13, where we have also added the value of the fitted straight lines for the strong and weak forcing from figure 11 at half the convective turnover time.

Clearly the effective viscosity increases for larger external forcing, but the effect is much smaller than the period dependence. Further, the perturbative calculation, which would correspond to a forcing strength of zero has a value at $T=\tau_{c} / 2$ of 0.055 , which is far below the range of direct calculation values obtained from either method. This is possibly 

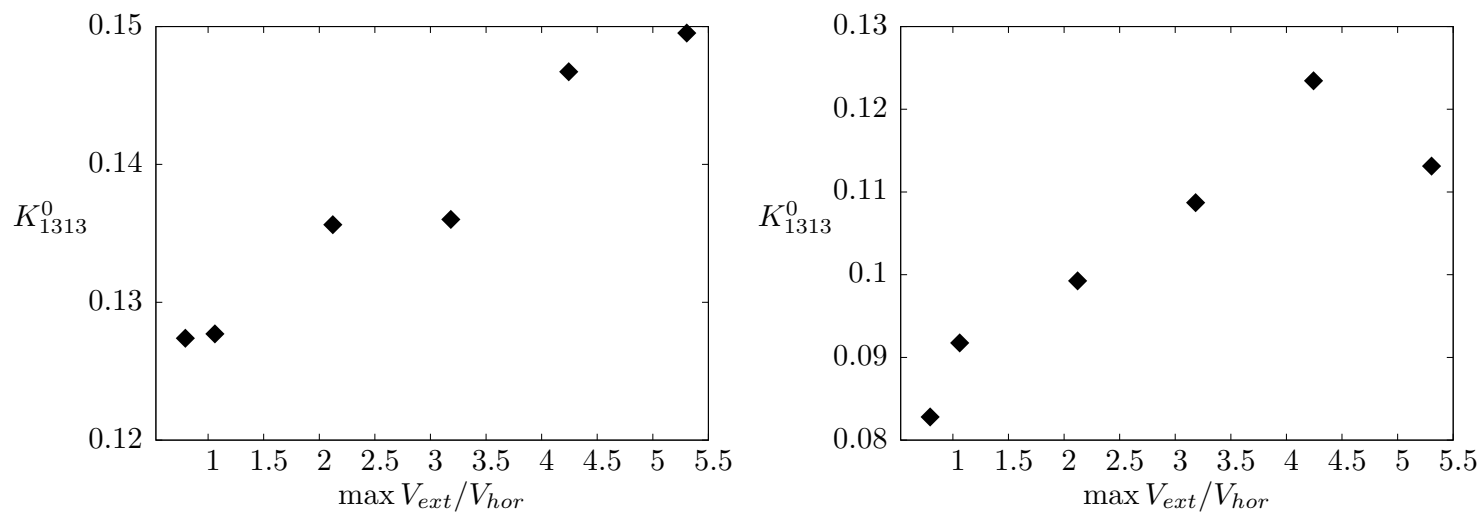

Fig. 13. - The dependence of the effective viscosity $K_{1313}^{0}$ on the forcing strength $\left(\max V_{\text {ext }} / V_{\text {hor }}\right)$, where $V_{\text {hor }}$ is the horizontal r.m.s. velocity in the absence of forcing. The left plot correspond to the effective viscosity estimated by least squares fitting of $W_{x z}^{\text {visc }}$ (equation 15) to $W_{x z}^{\text {turb }}$ (equation 14) and the right plot corresponds to the effective viscosity obtained by setting $\int W_{x z}^{\text {turb }}(z)=\int \widetilde{W}_{x z}^{\text {visc }}(z)$

due to the fact that at the periods covered by our direct calculation points the value of $T / \tau_{c}$ is not small enough for the perturbative calculation to be a good approximation. Another possibility is that for weaker forcing the amplitude dependence is much stronger, but we must note that according to the perturbative calculation, for very small amplitudes, the effective viscosity is independent of the amplitude of the external shear.

From figure 12 we see that this discrepancy between the direct calculation and the perturabative calculation does not exist for $K_{1212}^{0}$ and the weak forcing direct calculation points lie close to the the perturbative calculation curve.

A possible explanation for the observed amplitude dependence is that, at the forcing amplitudes we have used, the external shear is itself going to drive turbulence and lead to its own dissipation. Thus, the stronger the forcing, the more additional dissipation is expected to occur. 


\section{Conclusions}

We have completed a set of simulations of turbulent convection relevant to stellar surface convective zones, with external forcing introduced directly in the momentum equation in the form of a periodic, horizontal, position dependent gravitational acceleration. We then found an effective viscosity assumed to be of the form of equations 12 and 13 (in accordance with mixing length theory), using two methods:

1. least squares fitting of the work done by the external forcing on the flow at each plane of constant forcing to the energy transported or dissipated away from that plane by the effective viscosity.

2. matching the overall energy deposited into the box by the external forcing to the energy dissipated by the effective viscosity.

Both methods produce effective viscosity that scales linearly with period with the same slope, but they differ by a constant offset. This offset is due to the fact that, for most simulations, the negative side lobes of the energy removed by viscosity are less deep relative to the positive central peak than for the external work profile. For the least squares fitting method this tends to drive the dimensionless proportionality constant to higher values slightly over-predicting the central peak, but getting a bit deeper sidelobes. For the energy matching method, this tends to give a lower value for the viscosity in order to match the overall integrals under the curves.

This seems to suggest that the particular depth dependence we assumed for the turbulent viscosity might be slightly off. In particular it would seem to require a bit larger values at the depths of these side lobes.

Aside from this small effect, we found that the one parameter fits of the first method were able to capture the details of the observed position dependence of the deposited energy 
(see fig. 8, 9 and 10), which suggests that the effective viscosity assumption for treating turbulent dissipation in convective zones is valid.

We compared this directly obtained effective viscosity with the lowest order Goodman \& Oh (1997) perturbative expansion of Penev et al. (2008a), applied to the steady state flow without forcing, and we found that this method also predicts linear scaling of the effective viscosity with period, having a similar slope to the one predicted by the direct calculation.

Again there is a significant constant offset between the perturbatively calculated turbulent viscosity and the viscosity from the direct calculation. Some part of this offset might be due to the fact that the perturbative calculation is only valid when the external perturbation period is much smaller than the convective turnover time, and the smallest value we are able to simulate is $T \approx 0.3 \tau_{c}$.

At least part of this difference is due to actual amplitude dependence of the effective viscosity, which can be seen in figure 13 for $K_{1313}^{0}\left(\tau_{c} / 2\right)$. This amplitude dependence is possibly explained by the fact that the external shear caused by the introduced forcing is an additional driver of turbulence, and hence dissipation. While our calculations show clear evidence of this amplitude dependence, it is relatively less important than the period dependence at least in the range of amplitudes accessible to our numerical model.

The idea that the external shear is acting as an additional driver of turbulence suggest an explanation for why we might expect some extra viscosity at the location of the negative side lobes of the energy transfer curves. These regions correspond to the locations where the shear of the externally forced velocity field is the largest. Thus, if the external forcing is driving its own turbulence and causing its own dissipation, we would expect that extra viscosity to appear exactly at those locations. However, this explanation would suggest that the effect should decrease as the amplitude of the external forcing decreases and we see that 
this is not the case for the fixed period variable amplitude simulations, where the difference between the two methods seems more or less independent of the forcing amplitude.

To summarize, for forcing periods comparable to the convective turnover time $\left(\tau_{c}\right)$ the effective viscosity scales linearly with period and for our convective box its $K_{1313}$ and $K_{1212}$ components can be approximated by:

$$
\begin{aligned}
& K_{1313}(T) \approx \rho\left\langle v_{z}^{2}\right\rangle^{1 / 2} H_{p}\left[0.23 \min \left(\frac{T}{2.3 \tau_{c}}, 1\right)+\delta_{x z}(A)\right] \\
& K_{1212}(T) \approx \rho\left\langle\frac{v_{x}^{2}+v_{y}^{2}}{2}\right\rangle^{1 / 2} H_{p}\left[0.23 \min \left(\frac{T}{2.3 \tau_{c}}, 1\right)+\delta_{x y}(A)\right],
\end{aligned}
$$

where $T$ is the period of the external forcing, and $\delta_{x z}(A)$ and $\delta_{x y}(A)$ are constant amplitude dependent offsets that are also dependent on the particular method for deriving effective

\begin{tabular}{|c|c|c|c|c|}
\hline & \multicolumn{2}{|r|}{ Strong Forcing } & \multicolumn{2}{|r|}{ Weak Forcing } \\
\hline & fitted & dissipation matching & fitted & dissipation matching \\
\hline$\delta_{x z}$ & 0.10 & 0.06 & 0.08 & 0.03 \\
\hline$\delta_{x y}$ & 0.09 & 0.04 & 0.02 & -0.02 \\
\hline
\end{tabular}
viscosities:

The limited spatial resolution of our box does not allow us to reliably simulate the case of $T \ll \tau_{c}$. In that regime, the assumptions for Kolmogorov turbulence hold and the effective viscosity should scale quadratically with period (Goodman \& Oh 1997).

We compare the above directly calculated values to a perturbative estimate of the effective viscosity, which also predicts linear period dependence with the same slope, but with $\delta=0$ for all viscosity components.

The viscosity of equations 24 and 25 is closer to the Zahn (1966, 1989) prescription including the saturation period. However, the fact that we see the dissipation disappearing sharply at short periods suggests that the Zahn picture in which the dissipation is dominated 
by the largest eddies present is not the correct one. If this were the case the linear scaling seen at long periods should continue to hold for arbitrarily high frequencies. Instead our results suggest that it is the eddies with turnover times cloes to the external forcing period that cause most of the dissipation, and the observed linear scaling is due to the shallower than Kolmogorov time spectrum of the convective velocities. As a result, we expect the linear loss of efficiency to apply only in a limited range and for shorter periods faster loss of efficiency should occur. This might be the resolution of the apparent discrepancy between the dissipation necessary to explain tidal circularization and the red edge of the Cepheid instability strip on one hand and the observed amplitudes of the solar $p$-modes on the other. For small periods (of order minutes) the Kolmogorov scaling of turbulence holds and a quadratic decrease in the effective viscosity is appropriate. For long periods (of order days) the assumptions necessary for Kolmogorov cascade are not satisfied and the effective viscosity is found to scale linearly with period. 


\section{REFERENCES}

Goldreich, P. \& Keeley, D. A. 1977, ApJ, 211, 934

Goldreich, P. \& Kumar, P. 1988, ApJ, 326, 462

Goldreich, P., Murray, N., \& Kumar, P. 1994, ApJ, 424, 466

Goldreich, P. \& Nicholson, P. D. 1977, Icarus, 30, 301

Gonczi, G. 1982, A\&A, 110, 1

Goodman, J. \& Oh, S. P. 1997, ApJ, 486, 403

Malagoli, A., Cattaneo, F., \& Brummell, N. H. 1990, ApJ, 361, L33

Meibom, S. \& Mathieu, R. D. 2005, ApJ, 620, 970

Penev, K., Barranco, J., \& Sasselov, D. 2008a, ArXiv e-prints

Penev, K., Sasselov, D., Robinson, F., \& Demarque, P. 2007, ApJ, 655, 1166

—. 2008b, ArXiv e-prints

Robinson, F. J., Demarque, P., Li, L. H., Sofia, S., Kim, Y.-C., Chan, K. L., \& Guenther, D. B. 2003, MNRAS, 340, 923

Sofia, S. \& Chan, K. L. 1984, ApJ, 282, 550

Stein, R. F. \& Nordlund, A. 1989, ApJ, 342, L95

Verbunt, F. \& Phinney, E. S. 1995, A\&A, 296, 709

Zahn, J. P. 1966, Ann. d'Astrophys., 29, 489

Zahn, J. P. 1989, A\&A, 220, 112 
This manuscript was prepared with the AAS IATEX macros v5.2. 